\title{
INDUCED GOOD GRADINGS OF STRUCTURAL MATRIX RINGS
}

\author{
JOHN DEWITT AND KENNETH L. PRICE
}

\begin{abstract}
Our approach to structural matrix rings defines them over preordered directed graphs. A grading of a structural matrix ring is called a good grading if its standard unit matrices are homogeneous. For a group $G$, a $G$-grading set is a set of arrows with the property that any assignment of these arrows to elements of $G$ uniquely determines an induced good grading. One of our main results is that a $G$-grading set exists for any transitive directed graph if $G$ is a group of prime order. This extends a result of Kelarev. However, an example of Molli Jones shows there are directed graphs which do not have $G$-grading sets for any cyclic group $G$ of even order greater than 2 . Finally, we count the number of nonequivalent elementary gradings by a finite group of a full matrix ring over an arbitrary field.
\end{abstract}

Many important results concerning gradings on rings and other algebraic structures have appeared over the last two decades. Some of these are very essential results that can serve as a guide for further study. For example, if a grading of a Lie algebra is by a semigroup, then the semigroup must be an abelian group (see [12, Proposition 3.3]). Fine gradings, good gradings, and elementary gradings are the most fundamental types of gradings on full matrix algebras (see [2] and [12]). For finite semigroups the good gradings of full matrix algebras can be placed in one-to-one correspondence with simple and 0-simple primitive factors of the semigroup (see [7]). For torsion-free groups, gradings with finite support of full matrix algebras are elementary gradings (see [21]).

More recent work has resulted in a classification of gradings for many classical simple Lie algebras (see [1], 8], [12] and [13]). A method to induce good gradings on Lie superalgebras, Lie algebras, and matrix algebras using directed graphs was developed in [5], [10, [11], and [20]. An analogous method to induce good and elementary gradings on incidence rings was introduced in [14] and extended in [18]. With an increased understanding of how to induce gradings, we can count the number of gradings of a particular type. For full matrix algebras, fine gradings are counted in [13] and elementary gradings are counted in [5].

Throughout this note, the term "ring" or "subring" will only be used for an associative ring with unity. If we specify additive notation for a group operation, which we will always use when we assume the group is abelian, then the identity element will be denoted by 0 . Otherwise we use multiplicative notation and the identity element will be denoted by 1 .

Suppose $G$ is a group and $R$ is a ring. We say $R$ is a $G$-graded ring if there is a direct sum $R=\bigoplus_{a \in G} R_{a}$, as a group under the addition of $R$, such that $R_{b} R_{c} \subseteq R_{b c}$ for all

Date: October 26, 2018.

2000 Mathematics Subject Classification. Primary 16W50; Secondary 16S50.

Key words and phrases. Graded Algebra, Directed Graph. 
$b, c \in G$. The subgroups $R_{a}, a \in G$, are called the homogeneous components and the elements of $\cup_{a \in G} R_{a}$ are called the homogeneous elements. The support of $S$ is the set $\operatorname{Supp}_{G} S=\left\{g \in G: S_{g} \neq 0\right\}$.

We define good and elementary gradings of structural matrix rings in Section 1. All of our results concern a specific type of good grading, which we call an induced good grading. As explained in Theorem 1.6, for a finite abelian group $G$ the number of induced gradings divides $|G|^{s}$, where $s$ is the number of arrows that are not loops of the directed graph. The construction of induced good gradings was used in [20] to count induced good gradings of certain structural matrix rings. These results also give the number of induced good gradings of certain kinds of incidence rings (see [14], [15], [18], and [19]).

In Section 2, we cover an example of a directed graph $J$, which is due to M. Jones (see [14]), that shows a structural matrix ring may have induced good gradings that are not elementary gradings. There are 13 vertices in $J$. If $G$ is cyclic of order $k$, then by Theorem 2.2 the number of induced good gradings of a structural matrix ring over $J$ is $k^{12}$ if $k$ is odd, $2 \cdot k^{12}$ if $k$ is even. In Section 2, we also prove Theorem 2.4, which extends a result of Kelarev [11, Theorem 2].

Suppose $S=\bigoplus_{a \in G} S_{a}$ and $T=\bigoplus_{a \in G} T_{a}$ are $G$-graded rings. A homomorphism of $G$ graded rings is a ring homomorphism $h: S \rightarrow T$ such that $h\left(S_{a}\right) \subseteq T_{a}$ for all $a \in \operatorname{Supp}_{G} S$. An isomorphism that is a homomorphism of $G$-graded rings is called an isomorphism of $G$ graded rings. In the case of matrix algebras there are gradings which are not good gradings but are isomorphic to good gradings (see [5, Example 1.3]). Isomorphic gradings for good group gradings of incidence algebras over partial orders have been studied by Miller and Spiegel (see [15]).

Section 3 is dedicated to counting the number of nonequivalent induced good gradings of a structural matrix ring. Theorem 3.6 provides a formula for the number of nonequivalent elementary gradings by a finite group of a full matrix ring over a field.

\section{Graded Structural Matrix Rings}

For an arbitrarily chosen ring $R$, we let $M_{n}(R)$ denote the full matrix ring on the set of square matrices over $R$. In [6] structural matrix rings are constructed using Boolean matrices. Instead we may use preordered directed graphs to define structural matrix rings as in Section 3.14 of [10]. However, we refer to directed edges as "arrows," which has the advantage of not overusing the term "edges" when referring to the underlying undirected graph (see [20]) or the edges of a Hasse Diagram, as in Section 2, It is also consistent with [9], which considers gradings of path algebras that are defined using directed graphs containing vertices and "arrows."

The directed graphs we consider have a finite number of vertices and no repeated arrows. Loops are allowed. The vertex set and the arrow set of a directed graph $D$ are denoted by $V(D)$ and $A(D)$, respectively. If there are $n$ vertices we may assume they are numbered so that $V(D)=\{1, \ldots, n\}$ and $A(D)$ is a subset of $V(D)^{2}=V(D) \times V(D)$. We often drop the parentheses and comma for any arrow $(v, w)$ and denote it simply by $v w$. In some 
cases it is more convenient to use lower case Greek letters as a notation to stand for the arrows of a directed graph.

For $a, b \in V(D)$ with $a b \in A(D)$ we let $E_{a b}$ denote the standard unit matrix, that is, $E_{a b}$ is the $n \times n$ matrix whose entry in row $a$ and column $b$ is 1 and all of its other entries are 0 . A matrix is blocked by $D$ if it is a linear combination of standard matrix units which are indexed by arrows of $D$. The subset of all blocked matrices in $M_{n}(R)$ is a free $R$-bimodule over $R$ and we denote it by $S(D, R)$.

Consider the product of two blocked standard unit matrices $B$ and $C$. We have $B C=0$ unless $B=E_{i j}$ and $C=E_{j k}$ for some $i, j, k \in V(D)$ such that $i j, j k \in A(D)$. Since $E_{i j} E_{j k}=E_{i k}$ we need $i k \in A(D)$. Therefore $S(D, R)$ is closed under multiplication if and only if $D$ is transitive. We say $D$ is reflexive if there is a loop at every vertex of $D$. If $D$ is preordered, i.e. reflexive and transitive, then $S(D, R)$ is a ring, which is called a structural matrix ring. Chapter 8 of [10] is devoted to gradings of matrix rings, with gradings of structural matrix rings considered in [10, Section 8.3].

Let $G$ be a group. Then $S=S(D, R)$ is $G$-graded if there is a direct sum $S=\bigoplus_{g \in G} S_{g}$, as a group under the addition of $S$. If $D$ is preordered, $S$ is a $G$-graded ring if $S_{g} S_{h} \subseteq S_{g+h}$ for all $g, h \in G$. A $G$-grading of $S(D, R)$ is called good if the standard unit matrices are homogeneous. We recall some definitions and notation from [20]. A transitive triple in $D$ is an ordered triple of vertices contained in

$$
\operatorname{Trans}(D)=\{(a, b, c): a, b, c \in V(D) \text { and } a b, b c, a c \in A(D)\} .
$$

A function $\Phi: A(D) \rightarrow G$ is a homomorphism if equation 1.1 holds for any $(a, b, c) \in$ Trans $(D)$.

$$
\Phi(a b) \Phi(b c)=\Phi(a c)
$$

A $G$-grading of $S=S(D, R)$ is induced by $\Phi$ if for $g \in G$, the homogeneous component $S_{g}$ is the span of all $E_{\alpha}$ with $\alpha \in A(D)$ such that $\Phi(\alpha)=g$. This is a good grading, which we call an induced good grading. This is not to be confused with the induced gradings defined by Bahturin and Zaicev [3, Definition 4.1], which are graded algebras formed by the tensor product of a graded algebra with an elementary graded matrix algebra in the case when their supports do not necessarily commute.

A homomorphism $\Phi: A(D) \rightarrow G$ is elementary if there are elements $g_{1}, \ldots, g_{n} \in G$ such that $\Phi(a b)=\left(g_{a}\right)^{-1} g_{b}$ for all $a, b \in V(D)$ such that $a b \in A(D)$. An elementary grading of $S$ is a grading induced by an elementary homomorphism. A subset $X$ of $A(D)$ is a $G$-grading set for $D$ if for every function $\phi: X \rightarrow G$ there exists a unique homomorphism $\Phi: A(D) \rightarrow G$ such that $\left.\Phi\right|_{S}=\phi$. See [20] for conditions on $D$ and $G$ that ensure all homomorphisms are elementary.

Remark 1.1. Denote the complete graph on $n$ vertices by $K_{n}=\{s t: 1 \leq s, t \leq n\}$. For any group $G$, a G-grading set for $K_{n}$ is given by $\{12,13, \ldots, 1 n\}$ (see, for example, [5], [1] and [20]). Given a homomorphism $\Phi: K_{n} \rightarrow G$, set $g_{i}=\Phi(1 i)$ for $i=1, \ldots, n$. For any $a, b \in G$, there is a transitive triple $(1, a, b)$ so equation 1.1 gives $\Phi(1 b)=\Phi(1 a) \Phi(a b)$. In other words, $\Phi(a b)=\left(g_{a}\right)^{-1} g_{b}$. Thus the induced good gradings of $M_{n}(R)=S\left(K_{n}, R\right)$ are just the elementary gradings. 
Notation 1.2. Suppose $D$ is a preordered directed graph and $G$ is a group.

(1) $\operatorname{Hom}(D, G)$ denotes the set of homomorphisms from $D$ to $G$.

(2) $C_{G}(D)$ denotes the cardinality of the set of distinct homomorphisms from $D$ to $G$, that is,

$$
C_{G}(D)=|\operatorname{Hom}(D, G)| .
$$

If all homomorphisms from $D$ to $G$ are elementary, then $C_{G}(D)=|G|^{n-1}$, where $n$ is the number of vertices of $D$. Similarly, if $X$ is a $G$-grading set for $D$, then $C_{G}(D)=|G|^{|X|}$. Sufficient conditions on the directed graph for a $G$-grading set to exist are provided in [14], [18], and [20]. Sufficient conditions on the group $G$ are provided in Theorem 2.4.

Example 1.3. Suppose $G$ is a group and $R$ is a $G$-graded ring such that $1 \in R_{1}$ and $R_{g} \neq 0$ for some $g \in G \backslash\{1\}$. We may view $R$ as a good $G$-graded structural matrix ring over $D$ with $V(D)=\{1\}$ and $A(D)=\{(1,1)\}$. There is a homomorphism $\Phi: A(D) \rightarrow G$ given by $\Phi(1,1)=1$. However, the grading of $R$ induced by $\Phi$ has only one homogenous component.

Remark 1.4. Example 1.3 shows there are good graded structural matrix rings whose gradings are not induced by homomorphisms. We note that this is incorrectly stated in [20, Remark 1.2(4)]. However, if $D$ is preordered and $R I \subseteq S_{1}$, where $I$ is the identity matrix, then the good grading of $S=S(D, R)$ can be induced by a homomorphism $\Phi$ defined so that $e_{a b} \in S_{\Phi(a, b)}$ for all $a, b \in V(D)$ with $a b \in A(D)$. This may be proved in the same way as [19, Theorem 2].

Definition 1.5. Let $D$ be a directed graph and let $G$ be an additively written abelian group. For every $\alpha \in A(D)$, we let $x_{\alpha}$ denote an unknown from $G$.

(1) The transitive triple equations is the homogeneous system of linear equations of the form

$$
x_{a b}-x_{a c}+x_{b c}=0
$$

for all $(a, b, c) \in$ Trans $(D)$ such that $a, b$, and $c$ are distinct vertices of $D$.

(2) Let $r$ denote the number of transitive triple equations and let $s$ denote the number of arrows that are not loops of $D$. The transitive triple equations lead to the matrix equation $A X=0$ where $A$ is an $r \times s$ integer matrix and $X$ is a vector of unknowns. We call $A$ a transitivity matrix for $D$ in $G$.

Theorem 1.6. Let $D$ be a directed graph and let $G$ be an abelian group. If $G$ is finite, then $C_{G}(D)$ divides $|G|^{s}$, where $s$ is the number of arrows that are not loops of $D$.

Proof. If there are no transitivity equations, then $C_{G}(D)=|G|^{s}$. Otherwise, $C_{G}(D)$ is equal to the number of solutions to $A X=0$, where $A$ is a transitivity matrix for $D$ in $G$. The solutions to $A X=0$ form a subgroup of $G^{s}$, so $C_{G}(D)$ divides $|G|^{s}$ by Lagrange's Theorem. 


\section{Abelian Grading Groups}

Structural matrix rings can have induced good gradings that are not elementary. We consider a particular directed graph, which we denote by $J$ since it was first studied by M. Jones (see [14]). The Hasse diagram for $J$ is shown in figure 1. The vertices are numbered from 1 to 13 . The edges all indicate arrows pointing up. Moreover, $J$ is preordered so there are loops at every vertex in addition to all of the arrows forced by transitivity.

Notation 2.1. For a finite group $G$, we set $\operatorname{ord}_{k}(G)=\left\{g \in G: g^{k}=1\right\}$ for $k \geq 1$.

Theorem 2.2. Suppose $R$ is a ring and $G$ is an additively written finite abelian group.

(1) If $G$ has odd order, then a $G$-grading set for $J$ is given by

$B=\{(3,10),(3,11),(3,12),(3,13),(2,4),(2,5),(3,6),(2,7),(3,7),(3,8),(1,9),(3,9)\}$.

In this case, every induced good $G$-grading of $S(J, R)$ is elementary and $C_{G}(J)=$ $|G|^{12}$.

(2) If $G$ has order 2, then $B \cup\{(1,8)\}$ is a $G$-grading set for $J$ and $C_{G}(J)=2^{13}$.

(3) If $G$ has even order greater than 2, then $C_{G}(J)=\left|\operatorname{ord}_{2}(G)\right| \cdot|G|^{12}$.

(4) If $G$ is cyclic of even order greater than 2, then $C_{G}(J)=2 \cdot|G|^{12}$. This is not a power of $|G|$, so there is no G-grading set for $J$.

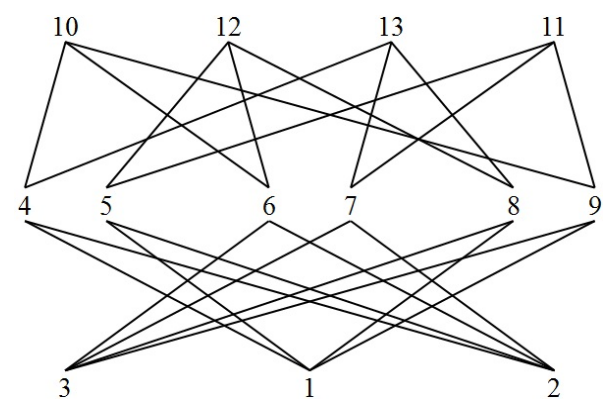

Figure 1. Hasse Diagram for J.

The transitive triple equations is a linear system of 24 equations in 36 unknowns. The transitivity matrix $A$ is shown in figure 2, Each column of $A$ corresponds to an arrow of $J$ and every row corresponds to a transitive triple. Every row contains two one's and a negative one, which come from the transitivity rule.

To solve the transitivity equations we can row-reduce $A$. Since we are looking for solutions in an abelian group we can only use integer row-operations. A row-echelon form of $A$ is shown in figure 3 . Each row has a leading entry in a column that corresponds to a dependent variable. The remaining variables are independent. Except for the last row, all of the entries are either 1,0 , or -1 . But in the last row every entry is a multiple of 2 . In a finite abelian group of even order there is more than one solution to the equation $2 x=0$. This leads to another independent variable to account for these solutions. 


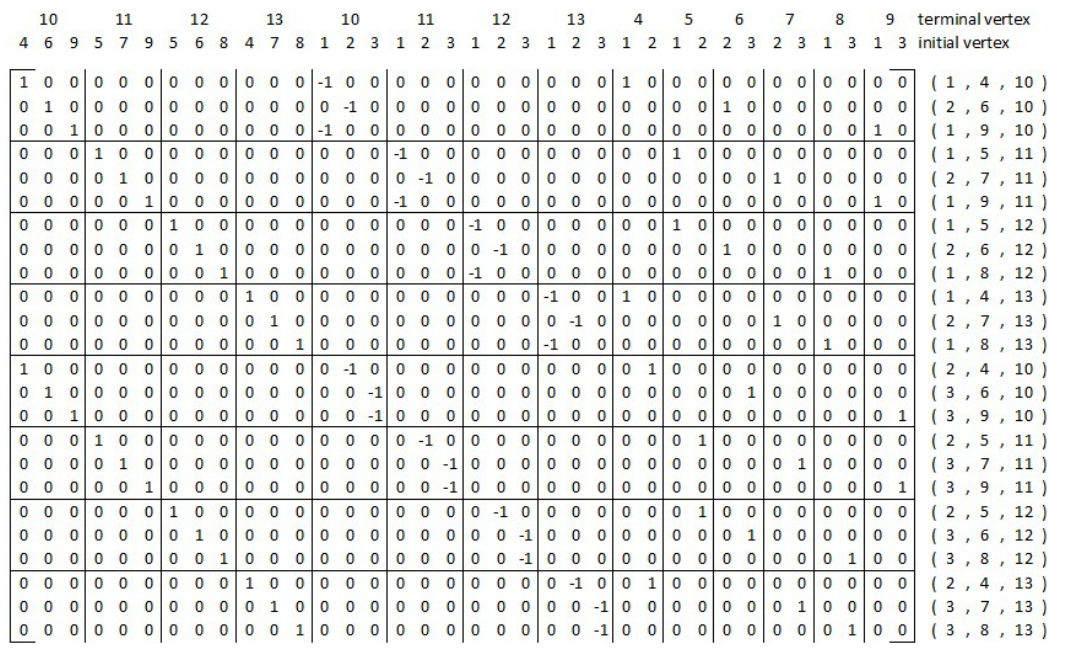

FIGURE 2. Transitivity Matrix for J.

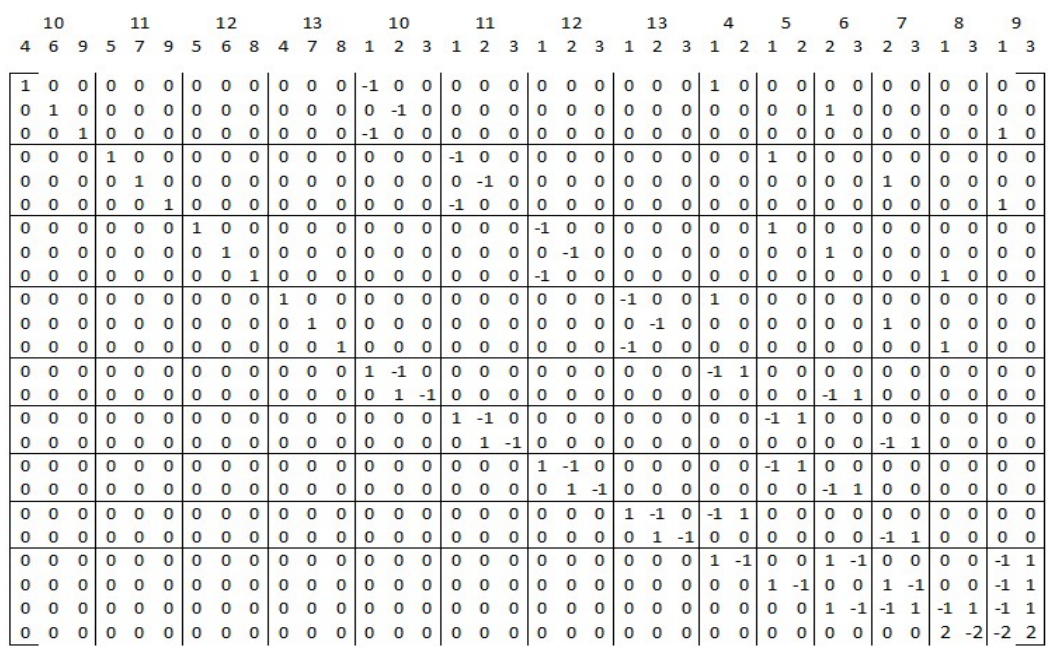

FiguRE 3. Row-Reduced Matrix

Suppose $G$ has odd order. In a parametric description the independent variables correspond to arrows $(3,10),(3,11),(3,12),(3,13),(2,4),(2,5),(3,6),(2,7),(3,7),(3,8)$, $(1,9)$, and $(3,9)$. We find this list of arrows from the row-echelon form of the matrix. This proves part 1 , since these are the only independent variables if the order of $G$ is odd. 
If $G$ has order 2, then the last row of the row-reduced matrix yields the equation $0=0$, so $x_{1,8}$ turns out to be an independent variable. Thus the set $B \cup\{(1,8)\}$ is a $\mathbb{Z}_{2}$-grading set for $J$. This proves part 2 .

If $G$ has even order then the last row of the row-reduced matrix yields solutions of the form

$$
x_{1,8}=x_{3,8}+x_{1,9}-x_{3,9}+T
$$

where the values of the independent variable $T$ are all of the elements of $G$ with order 1 or 2. This proves part 3. Part 4 follows easily from part 3.

Remark 2.3. Jones considered nonabelian group gradings over $J$ in [14, Example 6]. Her version of equation 2.1 is $x_{1,8}\left(x_{3,8}\right)^{-1} x_{1,9}\left(x_{3,9}\right)^{-1}=g$ or 1 , where $g$ is any element of $G$ with order 2.

A result of A. V. Kelarev (see [11, Theorem 2]) asserts that if $|G|=2$, then every finite and transitive directed graph has a $G$-grading set (which Kelarev calls a 'superbasis'). We furnish a new proof of this fact and extend the result to all finite groups of prime order.

Theorem 2.4. Suppose $D$ is a directed graph and $G$ is a finite group of order $p$, where $p$ is prime. Then there is a G-grading set for $D$ of size $s-r$, where $s$ is the number of arrows that are not loops of $D$ and $r$ is the rank of a transitivity matrix calculated over $\mathbb{Z} / p \mathbb{Z}$. Moreover, $C_{G}(D)=|G|^{s-r}$.

Proof. We may assume $G=\mathbb{Z} / p \mathbb{Z}$. Since $\mathbb{Z} / p \mathbb{Z}$ is a field under addition and multiplication modulo $p$ the transitivity matrix has a reduced row echelon form over $\mathbb{Z} / p \mathbb{Z}$. Thus there are $s-r$ independent variables ranging over $\mathbb{Z} / p \mathbb{Z}$ and $D$ contains a $\mathbb{Z} / p \mathbb{Z}$-grading set consisting of those arrows that correspond to independent variables.

Example 2.5. There are other directed graphs which do not contain G-grading sets if $G$ is cyclic of even order greater than 2, such as the one whose Hasse Diagram is shown in Figure 4. We do not know of any directed graphs which do not contain a G-grading set when $|G|$ is odd.

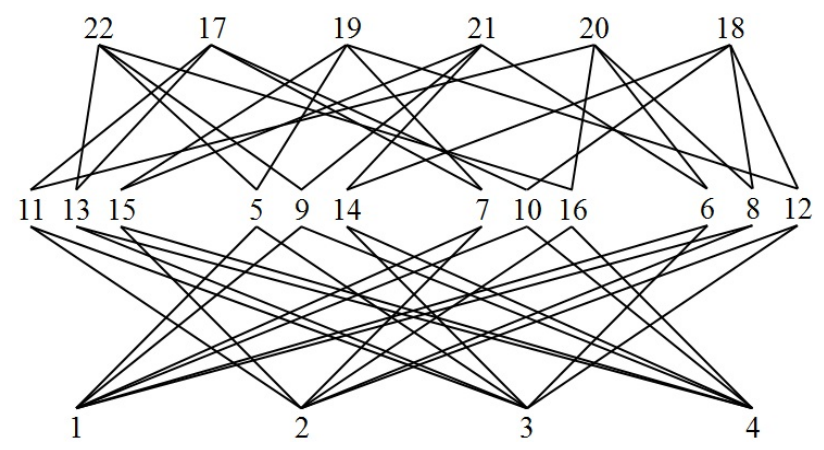

Figure 4. Another Hasse Diagram 


\section{Equivalent Good Gradings}

Definition 3.1. Suppose $D$ is a preordered directed graph, $G$ is a group, and $R$ is a ring. Set $S=S(D, R)$. We say that two G-gradings $S=\bigoplus_{g \in G} S_{g}$ and $S=\bigoplus_{g \in G} S_{g}^{\prime}$ of $S$ are equivalent if there is an automorphism of G-graded rings $h: S \rightarrow S$ such that $h\left(S_{g}\right) \subseteq S_{g}^{\prime}$ for all $g \in \operatorname{Supp}_{G} S$. Let $N_{G}(D, R)$ denote the cardinality of the set of nonequivalent induced good G-gradings of $S(D, R)$.

Example 3.2, which is due to Dăscălescu and van Wyk, shows that two structural matrix rings with good gradings may be graded isomorphic even if the underlying directed graphs are not isomorphic.

Example 3.2. By [6, Example 1.1], there is a ring $R$ such that

$$
\left[\begin{array}{cc}
R & 0 \\
0 & R
\end{array}\right] \cong\left[\begin{array}{cc}
R & R \\
0 & R
\end{array}\right] \cong\left[\begin{array}{cc}
R & 0 \\
R & R
\end{array}\right] \cong\left[\begin{array}{ll}
R & R \\
R & R
\end{array}\right] \cong R
$$

Suppose $S$ is one of the above $2 \times 2$ structural matrix rings that has nonzero off-diagonal entries. Then the underlying directed graph of $S$ contains an arrow $\alpha$ which is not a loop. If $g$ is a nontrivial element of a multiplicatively written group $G$, then there is a good $G$ grading on $S$ such that $E_{\alpha} \in S_{g}$. Using the isomorphism $S \cong R$ we obtain a good grading of $R$ such that $S$ and $R$ are isomorphic as $G$-graded rings.

Next we recall a few well-known terms from directed graph theory.

Definition 3.3. Let $D, D_{1}$, and $D_{2}$ be directed graphs.

(1) $D_{1}$ and $D_{2}$ are isomorphic if there is a bijection $\sigma: V\left(D_{1}\right) \rightarrow V\left(D_{2}\right)$ such that

$$
A\left(D_{2}\right)=\left\{(\sigma(a), \sigma(b)): a, b \in V\left(D_{1}\right) \text { and } a b \in A\left(D_{1}\right)\right\} .
$$

(2) The map $\sigma$ in part 1 is called an isomorphism.

(3) The arrow correspondence is the bijection $\sigma^{*}: A\left(D_{1}\right) \rightarrow A\left(D_{2}\right)$ given by $\sigma^{*}(a b)=$ $\sigma(a) \sigma(b)$ for all $a, b \in V\left(D_{1}\right)$ such that $a b \in A\left(D_{1}\right)$.

(4) The automorphism group of $D$, denoted by Aut $(D)$, is the group formed by all isomorphisms from $D$ to $D$ under composition.

Remark 3.4. Suppose $D$ is a directed graph and $G$ is a group. There is a group action of $\operatorname{Aut}(D)$ on $\operatorname{Hom}(D, G)$ given by $\sigma . \Phi=\Phi \circ\left(\sigma^{*}\right)^{-1}$ for any homomorphism $\Phi: D \rightarrow G$ and $\sigma \in \operatorname{Aut}(D)$. Let $\operatorname{Hom}(D, G)_{\sigma}$ be the set of all $\Phi \in \operatorname{Hom}(D, G)$ such that $\Phi \circ \sigma^{*}=\Phi$. If $G$ is finite, then by the orbit counting formula the number of orbits is given by

$$
|\operatorname{Hom}(D, G) / \operatorname{Aut}(D)|=\frac{1}{|\operatorname{Aut}(D)|} \sum_{\sigma \in \operatorname{Aut}(D)}\left|\operatorname{Hom}(D, G)_{\sigma}\right| .
$$

The problem of counting the number of fine gradings of a full matrix algebra over an algebraically closed field of characteristic zero was solved in [13. We will provide a formula that gives the number of nonequivalent elementary gradings by a finite group of any full matrix ring over a field $\mathbb{k}$. By remark 1.1, this is equal to the number of nonequivalent induced good $G$-gradings on the full matrix ring $S\left(K_{n}, \mathbb{k}\right)=M_{n}(\mathbb{k})$, which is $N_{G}\left(K_{n}, \mathbb{k}\right)$. We list the formulas for $n \leq 6$ below. These all follow from Theorem 3.6 . 
- $N_{G}\left(K_{2}, \mathbb{k}\right)=\frac{1}{2 !}\left(|G|+\left|\operatorname{ord}_{2}(G)\right|\right)$

- $N_{G}\left(K_{3}, \mathbb{k}\right)=\frac{1}{3 !}\left(|G|^{2}+3|G|+2\left|\operatorname{ord}_{3}(G)\right|\right)$

- $N_{G}\left(K_{4}, \mathbb{k}\right)=\frac{1}{4 !}\left(|G|^{3}+6|G|^{2}+8|G|+3\left|\operatorname{ord}_{2}(G)\right||G|+6\left|\operatorname{ord}_{4}(G)\right|\right)$

- $N_{G}\left(K_{5}, \mathbb{k}\right)=\frac{1}{5 !}\left(|G|^{4}+10|G|^{3}+35|G|^{2}+50|G|+24\left|\operatorname{ord}_{5}(G)\right|\right)$

- $N_{G}\left(K_{6}, \mathbb{k}\right)=\frac{1}{6 !}\left(|G|^{5}+15|G|^{4}+85|G|^{3}+210|G|^{2}+144|G|\right.$ $\left.+15\left|\operatorname{ord}_{2}(G)\right| \cdot|G|^{2}+90\left|\operatorname{ord}_{2}(G)\right| \cdot|G|+40\left|\operatorname{ord}_{3}(G)\right| \cdot|G|+120\left|\operatorname{ord}_{6}(G)\right|\right)$

Theorem 3.5. Let $\mathbb{k}$ be a field, let $G$ be a group, and let $n$ be a positive integer. Suppose $S=M_{n}(\mathbb{k})$ and $T=M_{n}(\mathbb{k})$, respectively, have $G$-gradings induced by homomorphisms $\Phi_{1}$ and $\Phi_{2}$, respectively. Then $S$ is graded isomorphic to $T$ if and only if there exists $\sigma \in \operatorname{Aut}\left(K_{n}\right)$ such that $\Phi_{2}=\Phi_{1} \circ \sigma^{*}$.

Proof. By Remark 1.1, $\Phi_{1}$ and $\Phi_{2}$ are elementary homomorphisms so there exist $g_{1}, \ldots, g_{n} \in$ $G$ and $h_{1}, \ldots, h_{n} \in G$ such that $\Phi_{1}(a b)=\left(g_{a}\right)^{-1} g_{b}$ and $\Phi_{2}(a b)=\left(h_{a}\right)^{-1} h_{b}$ for all $a, b \in V\left(K_{n}\right)$. Suppose $S$ is graded isomorphic to $T$. By the same argument in the proof of [4, Theorem 6], there exists $g_{0} \in G$ and $\sigma \in \operatorname{Aut}\left(K_{n}\right)$ such that $h_{i}=g_{0} g_{\sigma(i)}$ for all $i \in V\left(K_{n}\right)$. An easy calculation shows $\Phi_{2}(a b)=\Phi_{1}\left(\sigma^{*}(a b)\right)$ for all $a, b \in V\left(K_{n}\right)$, as desired. The reverse implication is straightforward.

We partition $S_{n}$, the permutation group on $n$ letters, following the construction and notation of principal characteristic polynomials described in [16. The cycle structure of a permutation $\sigma \in S_{n}$ is an $n$-tuple $\alpha=\left(\alpha_{1}, \ldots, \alpha_{n}\right)$ such that $\sigma$ is a (unique) product of disjoint $\alpha_{1}$ cycles on one letter, $\alpha_{2}$ cycles on two letters (i.e. transpositions), $\alpha_{3}$ cycles on three letters (i.e. ternary cycles), and so on.

We set $|\alpha|=\alpha_{1}+\cdots+\alpha_{n}$ and $d(\alpha)=\operatorname{gcd}\left\{i: \alpha_{i}>0\right\}$. If $\sigma \in S_{n}$ has cycle structure $\alpha$, then $\alpha_{1}+2 \alpha_{2}+\cdots+n \alpha_{n}=n$. Thus $d(\alpha)$ divides $n$ since either $\alpha_{x}=0$ or $d(\alpha)$ divides $x$ for every $x \leq n$. Conversely, if $d$ divides $n$, then $d(\alpha)=d$ for $\alpha$ such that $\alpha_{d}=\frac{n}{d}$. Let $P_{\alpha}$ denote the number of all permutations with cycle structure $\alpha$. An easy derivation of the formula for $P_{\alpha}$ given in equation 3.2 can be found in [16].

$$
P_{\alpha}=\frac{n !}{\left(1^{\alpha_{1}} \alpha_{1} !\right)\left(2^{\alpha_{2}} \alpha_{2} !\right) \cdots\left(n^{\alpha_{n}} \alpha_{n} !\right)}
$$

Theorem 3.6. Suppose $\mathbb{k}$ is a field and $G$ is a multiplicatively written finite group. The number of nonequivalent elementary $G$-gradings on $M_{n}(\mathbb{k})$ is given by

$$
N_{G}\left(K_{n}, \mathbb{k}\right)=\frac{1}{n !} \sum_{\alpha} P_{\alpha}\left|\operatorname{ord}_{d(\alpha)}(G)\right| \cdot|G|^{|\alpha|-1} .
$$

Proof. Note that $V\left(K_{n}\right)=\{1, \ldots, n\}$ and the automorphism group of $K_{n}$ is $S_{n}$. By Theorem 3.5 , equivalent induced $G$-gradings on $M_{n}(\mathbb{k})$ are the orbits under the action of $S_{n}$ on $K_{n}$. Therefore, using equation 3.1, we have

$$
N_{G}\left(K_{n}, \mathbb{k}\right)=\frac{1}{n !} \sum_{\sigma \in S_{n}}\left|\operatorname{Hom}\left(K_{n}, G\right)_{\sigma}\right| .
$$


By Remark 1.1, $B=\{12,13, \ldots, 1 n\}$ is a $G$-grading set for $K_{n}$ and $C_{G}\left(K_{n}\right)=|G|^{n-1}$. By [20, Theorem 2.3] and [20, Proposition 4.4], we may relabel the vertices and continue to use $B=\{12,13, \ldots, 1 n\}$ as a $G$-grading set for $K_{n}$. Moreover, two permutations $\sigma_{1}$ and $\sigma_{2}$ have the same cycle structure $\alpha$ if and only if they are conjugate. In this case, $\left|\operatorname{Hom}\left(K_{n}, G\right)_{\sigma_{1}}\right|=\left|\operatorname{Hom}\left(K_{n}, G\right)_{\sigma_{2}}\right|$. Thus it is enough to show equation 3.5 holds for any $\alpha$, where $\sigma$ is a permutation of cycle type $\alpha$.

$$
\left|\operatorname{Hom}\left(K_{n}, G\right)_{\sigma}\right|=\left|\operatorname{ord}_{d(\alpha)}(G)\right||G|^{|\alpha|-1}
$$

Case 1: If $\alpha=(n, 0, \ldots, 0)$ then $\sigma=$ id and $\left|\operatorname{Hom}\left(K_{n}, G\right)_{\sigma}\right|=\left|\operatorname{Hom}\left(K_{n}, G\right)\right|=$ $C_{G}\left(K_{n}\right)=|G|^{n-1}$. Therefore, equation 3.5 holds since $|\alpha|=n, d(\alpha)=1$ and $\operatorname{ord}_{d(\alpha)}(G)=\{1\}$.

In the remaining cases, $\sigma$ is a product of disjoint cycles $\sigma_{1}, \ldots, \sigma_{k}$ of length 2 or more, with $k \geq 1$. For $1 \leq i \leq k$, let $\ell_{i} \geq 2$ denote the length of cycle $\sigma_{i}$. Set $m_{0}=0, m_{1}=\ell_{1}$, $m_{2}=\ell_{1}+\ell_{2}, \ldots, m_{k}=\ell_{1}+\ell_{2}+\cdots+\ell_{k}$, and $m=m_{k}$. Without loss of generality, we may assume $\sigma_{i}$ sends $m_{i-1}+1$ to $m_{i-1}+2, m_{i-1}+2$ to $m_{i-1}+3$, etc., and $m_{i}=m_{i-1}+\ell_{i}$ to $m_{i-1}+1$ for $1 \leq i \leq k$. In particular, $\sigma(1)=\sigma_{1}(1)=2$.

We show equation 3.6 holds for all $i, j$ such that $1<i \leq k$ and $1<j \leq \ell_{i}$.

$$
\Phi\left(1, m_{i-1}+j\right)=\Phi(1,2)^{j-1} \Phi\left(1, m_{i-1}+1\right)
$$

The transitive triple $\left(1,2, m_{i-1}+j\right)$, the identity $\Phi \circ \sigma^{*}=\Phi$, and $\sigma^{*}\left(1, m_{i-1}+j-1\right)=$ $\left(2, m_{i-1}+j\right)$ are all used in the calculation below.

$$
\begin{aligned}
& \Phi\left(1, m_{i-1}+j\right)=\Phi(1,2) \Phi\left(2, m_{i-1}+j\right) \\
& \Phi\left(1, m_{i-1}+j\right)=\Phi(1,2) \Phi\left(1, m_{i-1}+j-1\right)
\end{aligned}
$$

Setting $j=2$ gives $\Phi\left(1, m_{i-1}+2\right)=\Phi(1,2) \Phi\left(1, m_{i-1}+1\right)$. Setting $j=3$ and substituting gives

$$
\Phi\left(1, m_{i-1}+3\right)=\Phi(1,2) \Phi\left(1, m_{i-1}+2\right)=\Phi(1,2)^{2} \Phi\left(1, m_{i-1}+1\right) .
$$

Continuing in this way, we arrive at equation 3.6 .

We have $\alpha_{2}+\cdots+\alpha_{n}=k$ since $\sigma$ is a product of disjoint cycles $\sigma_{1}, \ldots, \sigma_{k}$ of length at least 2. Moreover, $\alpha_{1}=n-m_{k}$ since $n-m_{k}$ is the number of vertices that are fixed by $\sigma$. This gives $|\alpha|=\left(n-m_{k}\right)+k$. There are two remaining cases to consider.

Case 2: If $n=m$, then $n=m_{k}, \alpha_{1}=n-m_{k}=0,|\alpha|=k$, and $d(\alpha)=$ $\operatorname{gcd}\left\{\ell_{1}, \ell_{2}, \ldots, \ell_{k}\right\}$. For $1<i \leq k$ and $j=\ell_{i}$, we show $\Phi(1,2) \in \operatorname{ord}_{\ell_{i}}(G)$ using the transitive triple $\left(1,2, m_{i-1}+1\right)$, the identity $\Phi \circ \sigma^{*}=\Phi, \sigma^{*}\left(1, m_{i-1}+\ell_{i}\right)=$ $\left(2, m_{i-1}+1\right)$, and equation 3.6 in the computation below.

$$
\begin{aligned}
\Phi\left(1, m_{i-1}+1\right) & =\Phi(1,2) \Phi\left(2, m_{i-1}+1\right) \\
\Phi\left(1, m_{i-1}+1\right) & =\Phi(1,2) \Phi\left(1, m_{i-1}+\ell_{i}\right) \\
\Phi\left(1, m_{i-1}+1\right) & =\Phi(1,2) \Phi(1,2)^{\ell_{i}-1} \Phi\left(1, m_{i-1}+1\right) \\
1 & =\Phi(1,2)^{\ell_{i}}
\end{aligned}
$$


Thus $\Phi(1,2) \in \cap_{i=1}^{k} \operatorname{ord}_{\ell_{i}}(G)$ and $\Phi(1,2) \in \operatorname{ord}_{\operatorname{gcd}\left\{\ell_{1}, \ell_{2}, \ldots, \ell_{k}\right\}}(G)$. By equation 3.6 . $\Phi$ is completely determined by $\Phi(1,2) \in \operatorname{ord}_{d(\alpha)}(G)$ and $\Phi\left(1, m_{1}+1\right), \ldots, \Phi\left(1, m_{k-1}+1\right) \in$ $G$. Equation 3.5 holds in this case with

$$
\left|\operatorname{Hom}\left(K_{n}, G\right)_{\sigma}\right|=\left|\operatorname{ord}_{d(\alpha)}(G)\right||G|^{k-1}=\left|\operatorname{ord}_{d(\alpha)}(G)\right||G|^{|\alpha|-1} \text {. }
$$

Case 3: If $n>m$, then $n>m_{k}, \alpha_{1} \neq 0, d(\alpha)=1$, and $\operatorname{ord}_{d(\alpha)}(G)=\{1\}$. We use the transitive triple $\left(1,2, m_{k}+1\right)$, the identity $\Phi \circ \sigma^{*}=\Phi$, and $\sigma^{*}\left(1, m_{k}+1\right)=$ $\left(2, m_{k}+1\right)$ in the calculation below.

$$
\begin{aligned}
\Phi\left(1, m_{k}+1\right) & =\Phi(1,2) \Phi\left(2, m_{k}+1\right) \\
\Phi\left(1, m_{k}+1\right) & =\Phi(1,2) \Phi\left(1, m_{k}+1\right) \\
1 & =\Phi(1,2)
\end{aligned}
$$

By equation 3.6, $\Phi$ is completely determined by $\Phi\left(1, m_{1}+1\right), \ldots, \Phi\left(1, m_{k-1}+1\right) \in$ $G$ and $\Phi\left(1, m_{k}+1\right), \Phi\left(1, m_{k}+2\right), \ldots, \Phi(1, n) \in G$. Equation 3.5 holds in this case with

$$
\left|\operatorname{Hom}\left(K_{n}, G\right)_{\sigma}\right|=|G|^{k-1}|G|^{n-m_{k}}=\left|\operatorname{ord}_{d(\alpha)}(G)\right||G|^{|\alpha|-1} .
$$

Remark 3.7. Suppose $n$ and $|G|$ are relatively prime. Recall $d(\alpha)$ divides $n$ for every $\alpha$. Thus $d(\alpha)$ and $|G|$ are relatively prime, which means $\operatorname{ord}_{d(\alpha)}(G)=\{1\}$. In this case formula 3.3 simplifies to formula 3.7 .

$$
N_{G}\left(K_{n}, \mathbb{k}\right)=\frac{1}{n !} \sum_{\alpha} P_{\alpha}|G|^{|\alpha|-1}
$$

Formula 3.8 shows Murnaghan's notation for $q_{n}$, the principal characteristic of $S_{n}$, where $s_{1}, s_{2}, \ldots, s_{n}$ are indeterminates (see [16]).

$$
q_{n}\left(s_{1}, s_{2}, \ldots, s_{n}\right)=\sum_{\alpha} \frac{1}{\alpha_{1} ! \alpha_{2} ! \cdots \alpha_{n} !}\left(\frac{s_{1}}{1}\right)^{\alpha_{1}}\left(\frac{s_{2}}{2}\right)^{\alpha_{2}} \cdots\left(\frac{s_{n}}{n}\right)^{\alpha_{n}}
$$

Then formula 3.7 becomes $N_{G}\left(K_{n}, \mathbb{k}\right)=q_{n}(|G|, \ldots,|G|) \cdot|G|^{-1}$.

As an application of Theorem 3.6, we offer Corollary 3.8, which is a well-known result for finite groups.

Corollary 3.8. Suppose $G$ is a finite group. Then

$$
\left|\operatorname{ord}_{p}(G)\right| \equiv|G|^{p-1}(\bmod p)
$$

for any prime number $p$.

Proof. Setting $n=p$ and multiplying by $p$ ! on both sides of equation 3.3 gives

$$
(p !) N_{G}\left(K_{p}, \mathbb{k}\right)=\sum_{\alpha} P_{\alpha}\left|\operatorname{ord}_{d(\alpha)}(G)\right| \cdot|G|^{|\alpha|-1} .
$$

We show that reducing mod $p$ yields the result since all of the terms disappear except in cases 1 and 2 below. 
Case 1: If $\alpha=(p, 0, \ldots, 0)$, then $P_{\alpha}=1$ by formula 3.2 and $\left|\operatorname{ord}_{d(\alpha)}(G)\right| \cdot|G|^{|\alpha|-1}=$ $1 \cdot|G|^{p-1}$.

Case 2: If $\alpha=(0, \ldots, 0,1)$, then, by formula 3.2, $P_{\alpha}=(p-1) !$ and $(p-1) ! \equiv-1$ $(\bmod p)$ by Wilson's Theorem. Moreover, $\left|\operatorname{ord}_{d(\alpha)}(G)\right| \cdot|G|^{|\alpha|-1}=\left|\operatorname{ord}_{p}(G)\right| \cdot 1$.

Case 3: For $\alpha \notin\{(p, 0, \ldots, 0),(0, \ldots, 0,1)\}$, we have $\alpha_{1}, \ldots, \alpha_{p-1}<p$ and $\alpha_{p}=0$ so none of the terms in the denominator of formula 3.2 is a multiple of $p$. Thus $P_{\alpha} \equiv 0(\bmod p)$.

Acknowledgements. The authors express their gratitude to the referee. One of the authors, Price, dedicates his contributions to Dr. Jeffrey Bergen, who recently passed away.

\section{REFERENCES}

[1] Y. Bahturin, M. Kochetov, and A. Rodrigo-Escudero, Gradings on classical central simple real Lie algebras, J. Alg. 506 (2018), 1-42.

[2] Y. Bahturin, S. Sehgal, and M. Zaicev, Group Gradings on Associative Algebras, J. Alg. 241 (2001), 677-698.

[3] Y. Bahturin and M. Zaicev, Group Gradings on Matrix Algebras, Canad. Math. Bull. 45 (2002), 499-508.

[4] Y. Bahturin and M. Zaicev, Gradings of Simple Algebras of Finitary Matrices, J. Alg. 241 (2001), 677-698.

[5] S. Dăscălescu, B. Ion, C. Năstăsescu, and J. Rios Montes, Group Gradings on Full Matrix Rings, J. Alg. 220 (1999), 709-728.

[6] S. Dăscălescu and L. van Wyk, Do Isomorphic Structural Matrix Rings have Isomorphic Graphs?, Proc. Amer. Math. Soc. 124 (1996), 1385-1391.

[7] S. Dăscălescu, A. V. Kelarev, and L. van Wyk, Semigroup Gradings of Full Matrix Rings, Comm. Algebra 29(11) (2001), 5023-5031.

[8] A. Elduque and M. Kotchetov, Gradings on Simple Lie Algebras. Mathematical Surveys and Monographs, vol. 189, American Mathematical Society, Providence, RI, 2013.

[9] E. L. Green, Graphs with Relations, Coverings and Group-graded Algebras, Trans. Amer. Math. Soc., 279 (1983), 297-310.

[10] A. V. Kelarev, Ring Constructions and Application. World Scientific, River Edge, NJ, 2002.

[11] A. V. Kelarev, Directed Graphs and Lie Superalgebras of Matrices, J. Alg. 285 (2005), 1-10.

[12] M. Kotchetov, Gradings on Finite-Dimensional Simple Lie Algebras, Acta Appl. Math. 108 (2009), 101-127.

[13] M. Kotchetov, N. Parsons, and S. Sadov, Counting Fine Gradings on Matrix Algebras and on Classical Simple Lie Algebras, Internat. J. Algebra Comput. 22(7) (2013), 1755-1781.

[14] M. R. Jones, Elementary and good group gradings of incidence algebras over partially-ordered sets with cross-cuts, Comm. Algebra 34(7) (2006), 2369-2387.

[15] L. Miller and E. Spiegel, Group Gradings in Incidence Algebras, Comm. Algebra 38(3) (2010), 953-963.

[16] F. D. Murnaghan, On the Representations of the Symmetric Group, American Journal of Mathematics $\mathbf{5 9}(3)$ (1937), 437-488.

[17] M. M. Parmenter and E. Spiegel, Central Gradings of Incidence Algebras, Algebra Colloquium 11(4) (2004), 421-426.

[18] K. Price, Good Gradings of Generalized Incidence Rings, Comm. Algebra 41(10) (2013), 3668-3678.

[19] K. Price, Corrigendum: Good Gradings of Generalized Incidence Rings, Comm. Algebra 46(3) (2018), 1047. 
[20] K. Price and S. D. Szydlik, Good Gradings from Directed Graphs, Contemp. Math. 609 (2014), 267276.

[21] S. K. Segal and M. Zaicev, Finite Gradings of Simple Artinian Rings, Moscow Univ. Math. Bull. 56 (2001), 21-24.

Mathematics Department, Nathan Hale High School, 11601 West Lincoln Avenue, West Allis, WI 53227

E-mail address: dewittj@wawm.k12.wi.us

Department of Mathematics, University of Wisconsin Oshkosh, 800 Algoma Boulevard, OSHKOSH, WISCONSIN 54901

E-mail address: pricek@uwosh.edu 